\title{
Unique challenges and the changing face of oncology during the COVID-19 pandemic and beyond
}

\author{
Catherine S. Hwang', MD, MSPH, Charles L. Bennett ${ }^{2,3}$, MD, PhD, MPP, and David M. Aboulafia ${ }^{4,5, *}$, MD \\ 1Department of Medicine, Virginia Mason Medical Center, Seattle, WA, USA \\ ${ }^{2}$ College of Pharmacy, University of South Carolina, Columbia, SC, USA \\ ${ }^{3}$ Visiting Investigator, City of Hope Comprehensive Cancer Center, Duarte, CA, USA \\ ${ }^{4}$ Floyd and Delores Jones Cancer Institute, Virginia Mason Medical Center, Seattle, WA, USA \\ ${ }^{5}$ Division of Hematology, University of Washington School of Medicine, Seattle, WA, USA
}

The novel coronavirus disease of 2019 (COVID-19) has profoundly impacted nearly every facet of our daily lives. People living with cancer are among the most fragile, vulnerable, and at risk for serious and life-threatening complications related to COVID-19 [1]. In this commentary, we highlight the unique challenges cancer specialists and those living with cancer face amidst the COVID-19 pandemic, as well as the implications of these challenges on oncology care in the future.

People living with cancer and their families endure heavy and sometimes overwhelming physical, emotional, and psychological burdens. As physicians and health care professionals, physical touch and non-verbal gestures are integral tools that enable us to connect more deeply with our patients at a personal level. Whether through an encouraging smile, supportive nod, or caring hug, our non-verbal gestures often provide comfort and reassurance in times of hardship. Shrouded by personal protective equipment, separated by physical distance to the extent possible, and "examining" our patients through telemedicine, our interactions are now interlaced with caution as we endeavor to protect our patients' fragile immune systems. Once innocent and intimate patient encounters are now unfamiliarly detached and formal. Words and silence have suddenly become ever more powerful, as verbal expressions of empathy communicated through personal protective equipment or via telemedicine are far from adequate substitutes for our previously common non-verbal gestures of caring and compassion.

Adding to this, we must thoughtfully consider the individual circumstances of our patients to assess whether the benefits of coming to the hospital or clinic outweigh the risks, or whether care can be conducted via telemedicine. Unfortunately, the guidelines we currently employ are largely derived from intuition, as evidence supporting best practices at a given location is not necessarily generalizable across cancer centers. Given the potential for asymptomatic spread of COVID-19 in an already immunocompromised population at increased risk for infection-related complications, simple tasks, such as obtaining a parking ticket, interacting with symptom screeners at the hospital entrance, riding in an elevator, and walking through the hospital hallways, are not necessarily benign. Universal masking, hand sanitizer, and floor signage with 6-feet markings to encourage awareness about the importance of physical distancing contribute to hospital-wide efforts to help keep our patients safe. Despite this, our ever-changing understanding of COVID19 infection, the challenges and limitations of current viral testing and contact tracing, and the growing burden of morbidity and mortality associated with this contagion engender little confidence among our patients. The rapid adoption of telemedicine has enabled clinical teams to engage with patients remotely when appropriate and may be the new normal for some patients. However, this promising approach is imperfect, as older patients struggle with operating newer communication tools, those of lower socioeconomic status grapple with access to internet and synchronous audiovisual technologies, and patients and families are left to decipher non-verbal cues that are often difficult to transmit with these media. The negative impact of COVID-19 on continuity of care is evidenced by a recent study, which found a $56 \%$ decline in in-person visits and a more than two-fold increase in telemedicine visits among adults at the United States Department of Veterans Affairs during the first 10 weeks of the pandemic, corresponding to an overall $30 \%$ decrease in the number of outpatient visits [2].

*Corresponding author: David M. Aboulafia, MD, Floyd and Delores Jones Cancer Institute, Virginia Mason Medical Center, 1100 Ninth Avenue (C2-HEM), Seattle, WA 98101, United States. Email: David.Aboulafia@ virginiamason.org

Received 6 September 2020 Accepted 19 October 2020 Published 27 October 2020

Citation: Hwang CS, Bennett CL, Aboulafia DM. Unique challenges and the changing face of oncology during the COVID-19 pandemic and beyond. J Cancer Res Ther. 2020; 8(5):28-29. DOI: 10.14312/2052-4994.2020-5

Copyright: ( 2020 Hwang CS, et al. Published by NobleResearch Publishers. This is an open-access article distributed under the terms of the Creative Commons Attribution License, which permits unrestricted use, distribution and reproduction in any medium, provided the original author and source are credited. 
Many of our patients with cancer, formerly accompanied by loved ones and caregivers to clinic appointments and hospital admissions, now must endure these visits alone due to newly implemented visitor restriction policies. At a time of vulnerability when patients are at the greatest need for social support, physical distancing has tangibly increased their loneliness and sense of isolation. Some of the saddest circumstances are when patients choose to forego necessary treatment or when they die alone in an unfamiliar hospital setting. Although technologies cannot replace human presence and emotions that were previously shared at the bedside, proliferating synchronous audiovisual capabilities, such as Skype, FaceTime, and Zoom, are increasingly enabling family members and caregivers to virtually comfort patients at the bedside and participate in discussions that occur in the clinic and hospital.

Individuals actively undergoing cancer treatment face additional challenges. The lack of universal asymptomatic screening for COVID-19 prior to chemotherapy places patients with cancer and clinic staff at risk for complications and adverse events if they are unknowingly positive for COVID-19. In addition, delays in cancer screening, diagnostic workup, treatment initiation, and interval follow-up have engendered a shared sense of fear, anger, and anxiety among patients with cancer [3]. Furthermore, many new clinical trials for experimental cancer therapies have been postponed while many existing clinical trials have deviated from their a priori protocols in light of the COVID-19 pandemic [4]. Given that chemotherapy is a cornerstone of cancer treatment and experimental treatments available through participation in clinical trials are often last source of hope in cancer care, the COVID19 pandemic leaves many cancer specialists pondering how to best care for their patients amidst disruptions in clinical investigations. As testing supplies become more widespread, screening all patients with cancer for COVID19 prior to chemotherapy would aid cancer specialists in proactively re-scheduling chemotherapy if indicated. Yet, we acknowledge the imperfect sensitivities and specificities of current diagnostic tests, as well as the need for appropriate resource allocation in regions where testing supplies and personal protective equipment remain limited. Furthermore, developing strategies to safely continue clinical trials in oncology and make experimental therapies available to patients for compassionate use is essential in maintaining access to potentially beneficial treatments in cancer care.

The risks and consequences of COVID-19 exposure have intensified fear in many individuals, causing them to avoid the hospital when they need medical care and postpone important cancer screening tests. Many emergency rooms and hospitals around the country have been eerily quiet, owing to the substantial reductions in visits for conditions unrelated to COVID-19, including myocardial infarctions, pulmonary emboli, and strokes [5]. The lack of trust in the healthcare system with regard to who has been exposed to COVID-19 may contribute to this fear. People living with cancer already carry an increased risk for thrombotic disease because cancer is a hypercoagulable state. Coupled with a concurrent COVID-19 infection, the risk of thrombotic complications may be amplified [6]. Complications of cancer care, such as neutropenic fever and intractable nausea with dehydration, also necessitate immediate care. Timely treatment of certain medical conditions, such as myocardial infarctions and strokes, is imperative because it directly impacts long-term health outcomes and survival. Coordinated leadership at the local, state, and national levels is essential, both to reassure patients that it is safe to seek medical care and to accurately convey the risks of COVID-19 to the general public so that informed decisions around infection prevention can be supported. Implementing more robust strategies for timely screening for COVID-19 and providing medical staff with adequate personal protective equipment can increase protection while fostering safer interactions among both staff members and people living with cancer.

The challenges during the COVID-19 pandemic have catalyzed valuable opportunities for stakeholders to reexamine and develop approaches for optimizing access to and delivery of cancer care. The desperation to drive medical research forward in an era of uncertainty has led to the accelerated proliferation of published literature, sometimes with methodological limitations that make the results difficult to interpret [7]. The importance of judiciously considering the strengths and limitations of available data, often aided by the knowledge that an editor and multiple peer-reviewers have supported the publication, remains of paramount importance, as cancer care must continue to be guided by high quality evidence and the rigorous standards of the peer review process. As we adapt during the COVID-19 pandemic with some changes that may persist longitudinally, a shared sense of responsibility and solidarity will help sustain us in our efforts to support and advance cancer care, both during this pandemic and in the decades to follow.

\section{Acknowledgment}

We thank Virginia M. Green, PhD, for her editing expertise and assistance with manuscript preparation.

\section{Conflicts of interests}

The authors have no conflicts of interest to declare.

\section{References}

[1] Kuderer NM, Choueiri TK, Shah DP, Shyr Y, Rubinstein SM, et al. Clinical impact of COVID-19 on patients with cancer (CCC19): a cohort study. Lancet. 2020; 395(10241):1907-1918.

[2] Baum A, Kaboli PJ, Schwartz MD. Reduced In-Person and Increased Telehealth Outpatient Visits During the COVID-19 Pandemic. Ann Intern Med. 2020; M7320-3026.

[3] Gharzai LA, Resnicow K, An LC, Jagsi R. Perspectives on oncologyspecific language during the coronavirus disease 2019 pandemic: $A$ qualitative study. JAMA Oncol. 2020; 6(9): 1424-1428.

[4] Waterhouse DM, Harvey RD, Hurley P, Levit LA, Kim ES, et al. Early impact of COVID-19 on the conduct of oncology clinical trials and longterm opportunities for transformation: Findings from an American Society of Clinical Oncology Survey. JCO Oncol Pract. 2020; 16(7):417421.

[5] Mantica G, Riccardi N, Terrone C, Gratarola A. Non-COVID-19 visits to emergency departments during the pandemic: the impact of fear. Public Health. 2020; 183:40-41.

[6] Bikdeli B, Madhavan MV, Jimenez D, Chuich T, Dreyfus I, et al. COVID19 and thrombotic or thromboembolic disease: Implications for prevention, antithrombotic therapy, and follow-up: JACC State-of-theArt Review. J Am Coll Cardiol. 2020; 75(23):2950-2973.

[7] Robinson AG, Gyawali B, Evans G. COVID-19 and cancer: do we really know what we think we know? Nat Rev Clin Oncol. 2020; 17(7):386388. 